\title{
Extraction of Curcumin from Turmeric Powder Through Complexation
}

\section{SHERIN GEEVARGHESE THOMAS ${ }^{1 *}$ and KALLIKAT NARAYANAN RAJASEKHARAN ${ }^{2}$}

'Department of Chemistry, University College, Thiruvananthapuram 695581, India. ${ }^{2}$ Department of Chemistry, University of Kerala, Kariavattom 695581, India.

*Corresponding author E-mail: sherin@universitycollege.ac.in

http://dx.doi.org/10.13005/ojc/370325

(Received: May 11, 2021; Accepted: June 12, 2021)

\section{ABSTRACT}

The complexation behavior of curcumin with boron and oxalic acid to form rubrocurcumin is utilized for the extraction of curcumin from turmeric powder. The complex formation reaction has been hastened by the addition of a phase transfer catalyst. To recover curcumin from the complex, the decomposition of the rubrocurcumin is studied. It was found that ammonia solution is a better reagent for the recovery of curcumin from its boron complex. The complexation of curcumin and its recovery by decomposition do not adversely affect curcumin, as ascertained by spectroscopic methods. This new approach for extraction of curcumin affords its isolation in an appreciable yield and in lesser time.

Keywords: Curcumin, Isolation, Turmeric powder, Rubrocurcumin, Hydrolysis.

\section{INTRODUCTION}

Curcumin and the curcuminoids are rare examples of naturally occurring $\beta$-diketoneligands. Curcumin was isolated by Vogel and Pelletier ${ }^{1}$ in 1815. Several methods have been reported for the isolation of curcumin from turmeric, which include the petroleum ether extraction ${ }^{2}$, organic solvent extraction ${ }^{3}$, by treating the rhizomes with a mixture of isopropyl alcohol and hexane. In 2000, a method of treating the dried rhizomes with dichloromethane was reported in which ground rhizome was stirred in dichloromethane and heated at reflux for hours ${ }^{4}$. The microwave ${ }^{5,6,7}$ and ultrasound ${ }^{8}$ assisted extraction of curcumin has gained much attention during the onset of $21^{\text {st }}$ century. Later, Bagchi ${ }^{9}$ has reported the supercritical fluid extraction of curcumin from turmeric using different organic solvents. Also, the chemical composition of turmeric was reported as protein $(6.3 \%)$, fat $(5.1 \%)$, minerals $(3.5 \%)$, carbohydrates $(69.4 \%)$ and moisture (13.1\%) . Bimal Dutta ${ }^{10}$ reported that during the ethanolic extraction of curcumin yielded $125 \mathrm{mg}$ out of $100 \mathrm{~g}$ dried and powdered rhizomes. The curcumin content in turmeric was reported to be $3-6 \%$ of the weight of the rhizomes. ${ }^{11,12}$

The methods for the preparation of curcumin-boron complexes as reported by Spicer and Strickland ${ }^{13}$, as well as by Sui ${ }^{14}$ et al., are not only tedious, requiring very prolonged refluxing, but also use solvents such as benzene and pyridine.

This is an Open Access article licensed under a Creative Commons license: Attribution 4.0 International (CC- BY). Published by Oriental Scientific Publishing Company @ 2018

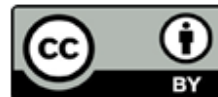


Thus it was felt that a short duration, less tedious, safer route to curcumin-boron complexes warrants investigation. With our long standing interest in the bioactivities of curcumin and curcumin-boron complexes, it was natural for us to consider whether the complex formation between boron and curcumin could be fashioned into a method for isolating curcumin from turmeric powder. Based on this idea, after a series of trials, we now report the optimized conditions for the complex formation and its subsequent decomposition to afford pure curcumin. In this context, the high current catalog price of pure curcumin, in comparison with the price of the same quantity of turmeric powder, which contains about $0.02-2 \%$ W/W curcumin, is noteworthy.

The formation of a deeply colored complex when curcumin was treated with boric acid or boric oxide has been known for more than a century. Later, this color formation was advantageously exploited for establishing a colorimetric method for the estimation of boron. The method is based on the formation of two different coloured complexes of curcumin with boron, which are rubocurcumin and rosocyanin. Between the two, the commonly used curcumin method for boron determination involves the formation of a reddish-brown rosocyanin complex ${ }^{13}$ with a $\lambda_{\max }$ of $545 \mathrm{~nm}$ at $\mathrm{pH} 1.0$. The chemistry behind the formation of rubrocurcumin and rosocyanin was investigated first by Spicer and Strickland ${ }^{14}$ who had suggested that rubrocurcumin is a 1:1:1 complex of curcumin, boron and oxalic acid whereas rosocyanin to be a 1:1 complex of curcumin with boron. Based on later work, including single crystal XRD studies of related compounds ${ }^{14}$, the structure of rubrocurcumin, the 1:1:1 complex of curcumin : boron : oxalic acid and rosocyanin, the 1:1 complex of curcumin : boron have been established.

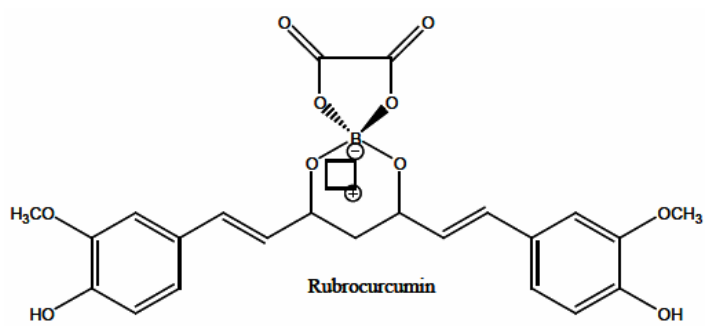

The metal complexes of curcumin were studied thoroughly for their structure and applications ${ }^{15}$. The presence of Boron in food and marine products was spectrophotometrically determined ${ }^{16,17}$ by reaction with curcumin. Thus the formation of rubrocurcumin as a new approach seems to be promising strategy in the extraction of curcumin rather than the other methods.

\section{MATERIALS AND METHODS}

In order to make the formation of rubrocurcumin easier, safer and more reproducible, several trials were carried out. The initial trials for the formation of rubrocurcumin were with dichloromethane, which we later replaced with ethyl acetate. This avoided the use of a chlorinated solvent, but the boron complex did not separate out so that the solvent had to be distilled to collect the complex. This prompted us to look for another eco-friendly solvent, from which the recovery of the product would be easy. Consequently, acetonitrile was examined as the reaction medium. In parallel, other organic carboxylic acids, such as malonic, succinic, lactic and tartaric acids were investigated with a view to tailor the solubility properties of the complex and thus enhance the yield of the complex. We however found that oxalic acid to be the best dicarboxylic acid among the acids studied.

Equimolar amounts of curcumin, boric acid and oxalic acid were thus mixed in acetonitrile and the mixture was heated for about an hour. However, the reaction did not reach completion by this time. Based on reports in literature, where phase transfer catalyst (PTC) speeded up the reaction in the organic phase, we attempted the inclusion of tetrabutylammonium bromide (TBAB) for hastening the complexation reaction ${ }^{18}$. The reaction was then seen to go to completion immediately as evidenced by the appearance of intense pink color on the addition of TBAB. Later, we noted that the addition of the PTC catalyst along with the reactants would reduce the overall heating time to less than ten minutes. The completion of the reaction could easily be confirmed by TLC analysis using chloroform and methanol (19:1). The work up involved a slow dilution of the reaction mixture with water under vigorous stirring whereupon the curcumin-boron-oxalic acid complex precipitated easily as a deep purple shiny micro crystals which are easily filterable. 
The recovery of curcumin from its boron complexes appears not to have been investigated in detail. The reported synthesis of curcumin ${ }^{19,20}$ involved a curcumin-boron complex as the final product from which curcumin had been reported to be released by treatment with dilute mineral or acetic acid and by stirring for several hours. To circumvent the above difficulty, we investigated many reagents that would make the recovery of curcumin easier. Taking cue from Pabon's report mentioned above, a saturated solution of rubrocurcumin in methanol was treated with dilute $\mathrm{NaOH}$ solution under stirring for $10 \mathrm{~min}$ at room temperature. Upon alkali addition and stirring, the color of the solution changed from blue to green, and finally to orange yellow. Upon acidification with dilute $\mathrm{HCl}$, the solution turned yellow. The mixture was extracted with ethyl acetate and the solvent was evaporated to obtain curcumin. However, this method cannot be adopted as a general method for the isolation due to the low solubility of rubrocurcumin in methanol and the instability of curcumin in presence of $\mathrm{NaOH}$. Moreover, an organic solvent was needed in this method to dissolve rubrocurcumin. Jeena et al., ${ }^{21}$ have studied the hydrolysis of rubrocurcumin in detail and utilized acetone-water medium for hydrolysis. When this was used as the medium for the decomposition of the boron complex, the recovery of curcumin was found to be difficult. In order to avoid the use of an organic solvent and also to make the recovery of curcumin operationally easier, ammonia solution was next investigated. Thus, rubrocurcumin was dissolved in concentrated ammonia and curcumin was easily recovered by further acidification.

Commercial turmeric powder $(50 \mathrm{~g})$ was mixed with oxalic acid $(2 \mathrm{~g})$ and boric acid $(2 \mathrm{~g})$ in $100 \mathrm{~mL}$ acetonitrile in a round bottom flask. The mixture was then heated for 10 minutes. After cooling, water was added followed by stirring to obtain a dark colored product. The product was then filtered and concentrated ammonia was added to the filter paper whereby the complex dissolved out, leaving the vegetable matter present in the turmeric powder in the filter funnel. The filtrate was diluted and neutralised with aqueous $\mathrm{HCl}$ solution to obtain curcumin in powder form upon stirring. It was then collected and the product was dried and weighed. The weight obtained was $1.36 \mathrm{~g}$. The product thus obtained was identified by TLC and by recording its VIS and FTIR spectra.
Table 1: Optimization of rubrocurcumin formation

\begin{tabular}{ccccc}
\hline Serial no & $\begin{array}{c}\text { Dicarboxylic acid } \\
\text { (in acetonitrile) }\end{array}$ & TBAB & Time & Yield \\
\hline \multirow{2}{*}{1} & & Yes & $10 \mathrm{~min}$ & $85 \%$ \\
& Oxalic acid & No & $60 \mathrm{~min}$ & $78 \%$ \\
& & Yes & $15 \mathrm{~min}$ & $60 \%$ \\
2 & Malonic acid & No & $75 \mathrm{~min}$ & $60 \%$ \\
& & Yes & $15 \mathrm{~min}$ & $60 \%$ \\
3 & Succinic acid & No & $75 \mathrm{~min}$ & $55 \%$ \\
& & Yes & $20 \mathrm{~min}$ & $50 \%$ \\
4 & Tartaric acid & No & $75 \mathrm{~min}$ & $55 \%$ \\
\hline
\end{tabular}

Dry column flash chromatography

Curcumin mix (500 mg) obtained by the decomposition of rubrocurcumin as above was dissolved in ethyl acetate. TLC grade silica $(5 \mathrm{~g})$ was added to it. Curcumin got adsorbed on the silica gel and the solvent was completely removed by evaporation at room temperature. A silica gel bed was packed in dry condition on a flash column. The adsorbed curcumin was then loaded above the silica bed of the dry column and the components were separated by dry column chromatography using elution with chloroform ${ }^{22}$ to obtain curcumin-I (370 $\mathrm{mg})$, curcumin-II (75 mg) and curcumin-III (30 mg).

\section{RESULTS AND DISCUSSION}

The formation of rubrocurcumin, which required about hours for completion under conditions reported by Spicer and Strickland, has now been made operationally much simpler by the use of a phase transfer catalyst TBAB and acetonitrile as solvent. The rapid, complete complex formation has been attributed to a heterogeneous phase transfer catalysis, though the exact mechanism of this catalysis has not been established. It has been suggested that the surface of the phase transfer catalyst serves as the site for reaction, whereas another reported explanation assumed that the small amount of moisture present aids the catalysis. In summary, acetonitrile seems to be a suitable solvent and the presence of TBAB was seen to be advantageous. Ammonia solution was found to most suited among a wide variety of acids and bases examined to decompose rubrocurcumin, owing to its inertness towards curcumin; unlike that of sodium hydroxide solution. Further, the precipitation and collection of curcumin powder by filtration or centrifugation is devoid of any practical difficulty. By employing this new method, we have been successful in isolating curcumin from the powdered rhizomes with an yield of $2.7 \%$. 
The hydrolysis study ${ }^{21}$ of a rubrocurcumin derivative from salicyclic acid showed that the end products are salicycilc acid and curcumin. In their study, the isolation of curcumin was confirmed based on UV-spectrum and HPLC analysis.

Rubrocurcumin neither undergoes any structural transformation during the treatment with $\mathrm{NH}_{3}$, nor curcumin underwent any unexpected structural changes during the decomposition as evidenced by TLC experiments. Also, the visible spectrum of rubrocurcumin in $\mathrm{NH}_{3}$ solution, followed by the treatment processes was similar to that of authentic curcumin. The $\lambda_{\max }$ value of curcumin falls around $430 \mathrm{~nm}$. Though curcumin has undergone complexation, dissolution and subsequent treatment with acid, the shape of the UV-spectrum as well as the $\lambda_{\max }$ value does not show any deviations from that of the reference sample. The uv spectrum was recorded at four different time intervals to ascertain that the curcumin isolated by the present method had not undergone any structural changes in presence of ammonia solution.

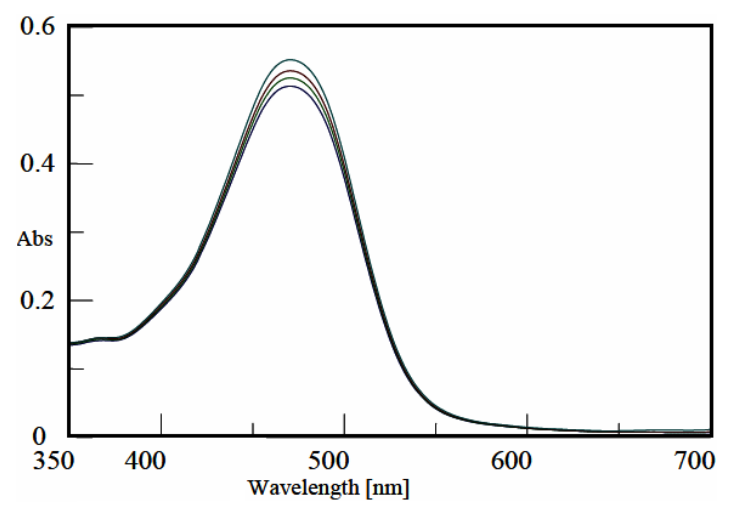

Fig. 1. Visible spectrum of curcumin in $\mathrm{NH}_{3}$ at $0,10,25$ and 30 minutes

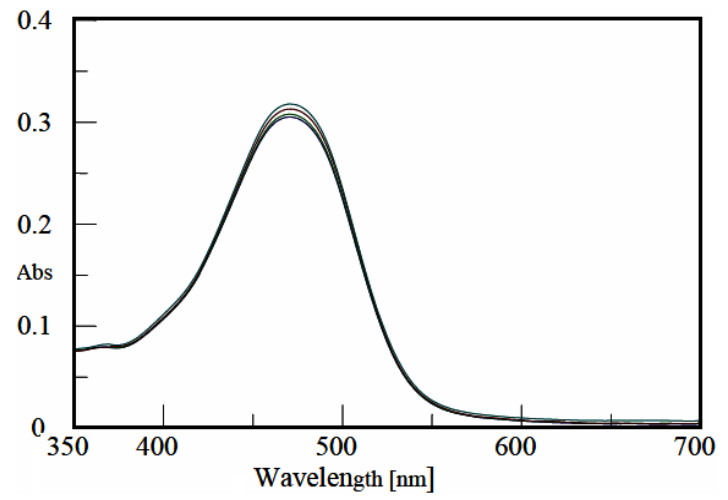

Fig. 2. Visible spectrum of rubrocurcumin in $\mathrm{NH}_{3}$ at $0,10,25$ and 30 minutes
The isolated curcumin powder was adsorbed on TLC grade silica and packed over the silica bed on a dry column. Three fine bands of the curcumin components can be visualized. This is due to the greater adsorbing capacity of TLC grade silica used in dry column flash chromatography, compared to that of the ordinary silica gel. Moreover, dry column flash chromatography is a method well adopted for the separation of components with minimum band gap in a column. The greater speed of the elution is noteworthy in the case of dry column flash chromatography.

\section{CONCLUSION}

Due to the presence of $\beta$-diketone moiety, curcumin is susceptible for complex formation with boron. This complexation behavior is utilized for the extraction of curcumin from turmeric powder. The literature reports available for the extraction of curcumin are invariably associated with the use of organic solvents in bulk amounts or else sophisticated methods such as SCF chromatography. Here, we report a method in which acetonitrile is employed for the complex formation reaction and a simple work up procedure. Moreover, rubrocurcumin undergoes decomposition on treatment with ammonia solution thereby releasing curcumin, thus leading to its isolation from turmeric powder. Our investigation ensured that the curcumin had not undergone any morphological or structural changes during the whole isolation process. The method affords the curcumin I-III mixture as a easily filterable powder within $30 \mathrm{~min}$, as against the 2-6 $\mathrm{h}$ required in reported, traditional extraction methods.

\section{ACKNOWLEDGEMENT}

The funding by Kerala State Council for Science Technology and Environment is acknowledged.

\section{Conflict of interest}

There is no conflict of interest regarding the publication of this article. 


\section{REFERENCES}

1. Vogel, H.; Pelletier, J.; Curcumin-Biological and Medicinal properties, Journal de Pharmacie, 1815, l, 289.

2. Janaki, N.; Bose, J. L., An improved method for isolation of curcumin from turmeric, Curcuma longa L., J. Indian Chem Soc., 1967 44, 985.

3. Harshal Ashok Pawar.; Amit Jagannath Gavasane and Pritam Dinesh Choudhary, A Novel and Simple Approach for Extraction and Isolation of Curcuminoids from Turmeric Rhizomes, Nat Prod ChemRes., 2018, 6, 1.

4. Anderson, A.; Mitchell, M.S.; Ram S. Mohan, Isolation of curcumin from turmeric, Journal of Chemical Education., 2000, 77(3), 359.

5. Mustafa Bene.; Mustafa Özyürek.; Kubilay Güçlü and Reşat Apak, Optimization of MicrowaveAssisted Extraction of Curcumin from Curcuma longa L. (Turmeric) and Evaluation of Antioxidant Activity in Multi-Test Systems, Rec. Nat. Prod., 2016, 10(5), 542-554.

6. Foozie Sahne.; Maedeh Mohammadi.;Ghasem D. Najafpour and Ali Akbar Moghadamnia, Extraction Of Bioactive Compound Curcumin From Turmeric (Curcuma Longa L.) Via Different Routes: A Comparative Study, Pak. J. Biotechnol., 2016, 13(3) 173-180.

7. Ghasem Najafpour.; Formic Acid And Microwave Assisted Extraction Of Curcumin From Turmeric (Curcumia Longa L), International Journal of Engineering., 2016.

8. Shital Patil.; R.C. Ranveer.; P.P. Debaje.; J.H. Kadam and A.K. Sahoo, Ultrasound Assisted Extraction of Curcumin, Asian Journal of Dairy and Food Research., 2018, 37, 250-252.

9. Bagchi, A.; Extraction of Curcumin, Journal of Environmental Sciences, Toxicology and Food Technology., 2012, 1(3), 01-16.

10. Bimal Dutta.; Study of secondary metabolite constituents and curcumin contents of six different species of genus Curcuma, Journal of Medicinal Plants Studies., 2015, 3(5), 116-119.

11. Ravindranath, V. and Satynarayana, M. N., An unsymmetrical diarylheptanoid from Curcumin longa, Phytochemistry., 1980, 19, 2031-2032.
12. Satyavati, G.V.; Raina, M.K. and Sharma M., Medicinal Plants of India, Indian Council of Medical Research., 1976, New Delhi, I.

13. Spicer, G.S. and Strickland, Studies on the compounds of curcumin with boric acid, Journal of Chemical Society., 1952, 4644-4650.

14. Sui, Z.; Salto, R.; Li, J.; Craik, C. and Paul, R. de M., Inhibition of HIV-I and HIV-II proteases by curcumin and curcumin boron complexes, Biorganic and Medicinal Chemistry., 1993, 1, 415-422.

15. Simon Wanninger.; Volker Lorenz.; Abdus Subhan, and Frank T. Edelmann, Metal complexes of curcumin - synthetic strategies, structures and medicinal applications, Chemical Society Reviews., 2015, 44, 4986-5002.

16. Liu, Y. \& Lee, K., Modifications of The Curcumin Method Enabling Precise And Accurate Measurement of Seawater Boron Concentration, Marine Chemistry., 2009, 115(1-2), 110-117.

17. Risna Erni Yati Adu.; Spectrophotometric Determination of Boron In Food Products By Ester Borate Distillation Into Curcumin, Journal of Chemistry., 2021, 67-73.

18. Dirgha Raj Joshi and Nisha Adhikari, Phase Transfer Catalyst in Organic synthesis, World Journal of Pharmaceutical Research., 2019, 8(8), 508-515. (Review Article).

19. Pabon H.J.J.; Synthesis of curcumin and related compounds, Recueil des Travaux Chimiques des Pays-Bas., 1954, 83, 379-382.

20. Roughley PJ.; Whiting DA. Experiments in the biosynthesis of curcumin. J. Chem. Soc Perkin I., 1973, 23, 79-88.

21. Jeena John.; Sudha Devi Rugmini and Balachandran Sreedharan Nair, Kinetic Analysis of Thermal and Hydrolytic Decomposition of Spiroborate Ester of Curcumin with Salicylic Acid, Orient. J. Chem., 2017, 33(2), 849-858.

22. Babu, K.V.D.B. and Rajasekhran K. N., Simplified conditions for the synthesis of curcumin I and other curcuminoids, Organic Preparations Procedure International., 1994, 26, 674. 